\title{
Dengue e saúde pública na cidade do Rio de Janeiro, Brasil
}

\author{
Bruna Sarpa Miceli \\ Graduação em Ciências Biológicas na Universidade Veiga de Almeida (UVA) \\ $\square$ brunasm213@gmail.com \\ Andresa Borges de Araujo Fonseca \\ Graduação em Ciências Biológicas na Universidade Veiga de Almeida (UVA) \\ $\triangle$ andresa.borges30@gmail.com
}

Recebido em 24 de novembro de 2017

Aceito em 21 de dezembro de 2017

\section{Resumo:}

Este presente artigo trata a questão do saneamento básico na cidade do Rio de Janeiro relacionandoo com a incidência de casos de dengue. Para isso, buscou-se trazer o conceito de saneamento básico segundo a Organização Mundial da Saúde (OMS) e também abordar historicamente a evolução desta concepção desde os primórdios do século XIX, com a ausência desse serviço, até o início do século XXI com o surgimento de leis de incentivo e de relatos do cenário atual principalmente com a infraestrutura da cidade durante o ano de 2016, quando ocorreram os Jogos Olímpicos, assim como pretende-se abordar informações referentes doença citada. 0 objetivo deste trabalho foi comparar os dados fornecidos pela Prefeitura da Cidade do Rio de Janeiro em relação ao saneamento básico (coleta de esgoto, com dados dos anos 2000 até 2014 e resíduos sólidos como o lixo público, referentes aos anos 2010 e 2014) com o número de casos de dengue (de 2010, 2014 e 2016) a fim de verificar o quanto a falta destes recursos interfere na qualidade de vida da população e no número de casos dessa enfermidade. Observando dados comuns de 2010 e 2014, notou-se que as questões de saneamento básico não condizem com os índices de casos de dengue nestes mesmos períodos. Conclui-se que não se pode relacionar tais dificuldades como causas únicas para a veiculação de doenças, principalmente da dengue e que há outros fatores que podem influenciar o aparecimento desta doença nas Regiões Administrativas (R.As).

Palavras-chave: Saneamento básico, Dengue, Infraestrutura, Saúde pública, Rio de Janeiro.

\section{Dengue and public health in Rio de Janeiro city, Brazil}

\begin{abstract}
:
This article deals with the issue of sanitation in the city of Rio de Janeiro relating with the incidence of cases of dengue fever. For that, we sought to bring the concept of basic sanitation, according to the World Health Organization (who) and also address the evolution of this concept historically since the early $19 \mathrm{TH}$ century, with the absence of that service, until the beginning of the 21st century with the emergence of incentive laws and reports of current scenario especially with the infrastructure of the city during the year 2016 When the Olympic Games, as well as a aims to address disease cited information. The aim of this study was to compare the data provided by the city of Rio de Janeiro in relation to basic sanitation (sewage collection, with data from the years 2000 until 2014 and solid waste as the public garbage for the years 2010 and 2014) with the number of cases of dengue (2010, 2014 and 2016) in order to check how the lack of these resources interferes with the quality of life of the population and in the number of cases that illness. Noting common data 2010 and 2014, it was noted that the issues of basic sanitation does not match the contents of dengue fever cases in these same periods. It appears that you can't relate to such difficulties as causes only
\end{abstract}


for airing of diseases, especially dengue and that there are other factors that can influence the appearance of this disease in the Administrative Regions (A.Rs).

Keywords: Sanitation, Dengue, Infrastructure, Public health, Rio de Janeiro.

\section{Dengue y la salud pública en la ciudad de Río de Janeiro, Brasil}

\section{Resumen:}

Este artículo aborda el tema de saneamiento en la ciudad de Río de Janeiro sobre la incidencia de casos de dengue. Por eso, intentamos acercar el concepto de saneamiento básico, según la Organización Mundial de la salud (OMS) y también la evolución de este concepto históricamente desde principios del siglo XIX, con la ausencia de ese servicio, hasta el comienzo del siglo XXI con la aparición de las leyes de incentivos y los informes de escenario actual especialmente con la infraestructura de la ciudad durante el año 2016 Cuando los Juegos Olímpicos, así como una enfermedad de dirección pretende cita información. El objetivo de este estudio fue comparar los datos proporcionados por la ciudad de Río de Janeiro, en lo referente a saneamiento básico (colección de las aguas residuales, con los datos de los años 2000 hasta 2014 y sólidos como la basura pública los desechos para los años 2010 y 2014) con el número de casos de dengue (2010, 2014 y 2016) para comprobar cómo la falta de estos recursos se interfiere con la calidad de vida de la población y el número de casos que enfermedad. Tomando nota de los datos comunes de 2010 y 2014, se observó que los problemas de saneamiento básico no coincide con el contenido de los casos de fiebre del dengue en estos mismos periodos. Parece que no relaciona estas dificultades como causas sólo para la transmisión de enfermedades, especialmente dengue y que existen otros factores que pueden influir en la aparición de esta enfermedad en las Regiones Administrativas (R.As).

Palabras clave: Saneamiento, Dengue, Infraestructura, Salud pública, Río de Janeiro.

\section{INTRODUÇÃO}

O conceito de saneamento básico é muito amplo, abrange diversos elementos e está totalmente interligado com a manutenção da saúde. Segundo a Organização Mundial da Saúde (OMS, 2017), este termo "refere-se à manutenção das condições de higiene através de serviços como a coleta e lixo e eliminação de águas residuais", ou seja, corresponde a um correto transporte, tratamento e despejo da água, aos serviços básicos que garantem a infraestrutura e limpeza do ambiente, ao controle e combate de doenças. Este significado era desconhecido décadas atrás e tampouco utilizado e presume-se que atualmente ainda haja falta destes recursos na sociedade, sendo capazes de interferir na qualidade de vida dos cidadãos e no meio ambiente (REIS, 2016).

\section{Abordagem histórica da infraestrutura da Cidade}

Apesar de ser algo imprescindível na vida da população, o saneamento nem sempre esteve presente na cidade do Rio de Janeiro. No século XVIII para XIX, o transporte de água 
e esgoto era realizado por escravos, sem nenhum tipo de tratamento ou infra-estrutura e postos de saúde pública eram inexistentes, ou seja, não havia preocupação com a saúde. Esta questão sanitária ganhou destaque devido aos problemas causados pela sua ausência tais como as epidemias de cólera e febre amarela (MARQUES, 1995; MIRANDA et al., 2013).

$\mathrm{O}$ ato de sanear, porém, ganhou força no século XX, a partir de melhorias e investimentos através da reforma urbana e da revolta da vacina, ocorrentes durante governo de Rodrigues Alves e a prefeitura de Pereira Passos cujas prioridades estavam relacionadas à modernização e ao saneamento (MIRANDA et al., 2013). Neste período, foram realizadas uma série de mudanças para promover a melhoria da cidade, entre elas o conhecido "Bota abaixo", que visava a limpeza e abertura de avenidas através da demolição de cortiços. Quanto às epidemias ainda existentes e o seu combate, estas ficaram sob a responsabilidade de Oswaldo Cruz, que implementou medidas de higiene e a criação da lei da vacinação obrigatória em 1904, resultando na revolta da população, mas também em um grande avanço na manutenção da saúde (PÔRTO e PONTE, 2003). Além disso, em 1916, surgiram os primeiros Postos de Higiene e Profilaxia Rural (PHPR) no Rio de Janeiro que visavam combater diversas enfermidades como malária e doença de Chagas. Em 1923, com a Reforma Carlos Chagas, houve a criação do Departamento de Saúde Pública (DSP) que permitiu um combate as endemias rurais e urbanas e um saneamento e higiene destas regiões (CAMPOS et al., 2016).

\section{A questão sanitarista dos anos 90 até os dias atuais}

A partir deste período, houve a criação de diversas leis que passaram a dispor sobre o saneamento e a saúde, como a Lei 8.080 de 19 de setembro de 1990, que em seu artigo $3^{\text {o }}$ afirma que "os níveis de saúde expressam a organização social e econômica do País, tendo a saúde como determinantes e condicionantes" além de outros serviços, como o saneamento básico (BRASIL, 1990). Mais adiante, a Lei 11.445 de janeiro de 2007, conhecida como a Lei do Saneamento Básico, estabelece que os serviços públicos de saneamento sejam oferecidos seguindo alguns princípios, tais como “a universalização do acesso; integralidade dos serviços de saneamento básico; abastecimento de água, esgotamento sanitário, limpeza urbana e manejo de resíduos sólidos sejam realizados de forma adequada à saúde pública e à proteção do meio ambiente; disponibilidade em todas as áreas urbanas", que torna o acesso 
à limpeza e à higiene um direito do cidadão (BRASIL, 2007). Complementando esta ideia, a Lei $n^{\circ} 12.305$ de 2 de agosto de 2010 estabelece a Política Nacional de Resíduos Sólidos, que está relacionada com a coleta seletiva e visa a destinação correta destes elementos e visa "gerenciamento ambientalmente adequado dos resíduos sólidos " (BRASIL, 2010).

Além da criação destas leis, atualmente, pode-se dizer que houve certo desenvolvimento para as questões relacionadas à infraestrutura, principalmente pelo fato da Cidade do Rio de Janeiro ser a sede dos Jogos Olímpicos no ano de 2016. Obras em diversos locais foram realizadas e entre elas, inclui-se o 'Porto Maravilha', 'TransOeste', 'Transolímplica' e entre outras.

Contrapondo a ideia supracitada, de acordo com Carvalho e Orsine (2011), o cenário atual está interligado com o aumento da industrialização e com o inadequado despejo de resíduos, resultando em grandes problemas ambientais. Complementando essa ideia, Lima (2006), afirma que o crescimento populacional intensificou os processos de poluição da água, especialmente no que diz respeito à Baía de Guanabara, no Rio de Janeiro, devido ao lançamento de esgotos sanitários e outros resíduos sólidos (FEEMA, 1998 apud LIMA, 2006) provocando fenômenos de eutrofização e tornando os serviços básicos oferecidos incombináveis com essa urbanização. Vale lembrar que estas redes de esgoto não tratadas oferecem diversos riscos para a população que, por sua vez, fica mais suscetível a contrair doenças transmitidas pela água, como a hepatite A, leptospirose, entre outras (REIS, 2016). Concomitantemente, Dornelles e Martins (2016) chamam a atenção para a problemática do Zika vírus, doença também transmitida pelo Aedes aegypti durante esse período, onde houve uma defasagem de notícias que relacionavam os jogos com essa doença.

\section{A dengue e seu histórico}

Sabe-se que assim como a febre amarela urbana, a dengue corresponde a mais uma doença infecciosa resultante do vírus do gênero Flavivirus que é transmitido principalmente pelo mosquito Aedes aegypti (CARVALHO et al., 2017). Wermelinger e Carvalho (2016) chamam a atenção para o surto de febre amarela durante o século XX e as tentativas de controle, uma delas, conforme já citado, engloba a vacinação obrigatória comandada por Oswaldo Cruz. Ainda segundo esses autores, nesta época havia, assim como é possível 
encontrar nos dias de hoje, alguns "condicionantes sociais e ambientais" auxiliadores da proliferação destes vetores como "ocupação desordenada e não planejada do espaço urbano, prioridade ou mesmo exclusividade dos serviços públicos básicos para a elite e carência ou ausência de saneamento básico para uma grande parcela da população" (WERMELINGER e CARVALHO, 2016).

Em relação à dengue, segundo Valle, Pimenta e Aguiar (2016), a partir de 1986, os casos de dengue passaram a ser cada vez mais ocorrentes e frequentes. Além disso, a mesma também está diretamente relacionada com a falta de saneamento e coleta, além do desordenado crescimento urbano (SILVA et al., 2014; TAUIL, 2001), contribuindo para o surgimento de criadouros do mosquito, principalmente quando se inclui o descarte inadequado de resíduos sólidos como garrafas de vidro ou plástico, copos, latas de alumínio, pneus. Por esse fato, ressalta-se ainda a necessidade de participação dos cidadãos quanto ao controle dessa enfermidade com simples ações que resultam na eliminação dos focos desses vetores "como evitar potenciais reservatórios de água em quintais, troca periódica da água de plantas aquáticas" além de garantir um correto descarte do lixo em locais apropriados, como as lixeiras.

Diante de tais fatos, é questionado se a insuficiência dos serviços básicos de saneamento na Cidade do Rio de Janeiro pode afetar a qualidade de vida da população e estar diretamente relacionados com os casos da dengue. Acredita-se que esta ineficiência seja capaz de contribuir para uma maior incidência desta doença, já que o município do Rio de Janeiro apresenta características bem heterogêneas, como diferenças sociais, econômicas e demográficas entre as regiões. (CARVALHO et al., 2017).

O objetivo deste trabalho foi comparar os dados fornecidos pela Prefeitura da Cidade do Rio de Janeiro em relação ao saneamento básico (coleta de esgoto, com dados dos anos 2000 até 2014 e resíduos sólidos como o lixo público, referentes aos anos 2010 e 2014) com o número de casos de dengue (de 2010, 2014 e 2016) a fim de verificar o quanto a falta destes recursos interfere na qualidade de vida da população e no número de casos dessa enfermidade. 


\section{METODOLOGIA}

Para esta pesquisa, foram analisados dados informacionais do site da Prefeitura da Cidade do Rio de Janeiro referentes à dengue (2010, 2014 e 2016), à coleta de esgoto (2000 até 2014), e lixo público (2010 e 2014). Estes dados foram relatados a fim de verificar se os serviços prestados no município do Rio de Janeiro ou em suas Regiões Administrativas (R.As) têm melhorado com o passar dos anos.

Em seguida, buscou-se fazer uma comparação do número de casos de dengue com estas questões de saneamento básico referentes ao ano de 2010 e 2014, comum nas 3 análises acima a fim de relacioná-los e observar se estas questões influenciaram no aumento de casos de dengue na Cidade.

\section{RESULTADOS}

Vale a pena ressaltar que a Cidade do Rio de Janeiro possui 34 R.As. A $32^{\text {a }}$ região administrativa não foi identificada no site fornecido pela Prefeitura do Rio de Janeiro, e desta forma, seguiu-se conforme o que foi disponibilizado, como pode ser observado na tabela abaixo (Tab.1) referente ao ano de 2010, juntamente com os bairros pertencentes de cada região e o número de habitantes.

Tabela 1 - Regiões administrativas do Rio de Janeiro com seus respectivos bairros, número de habitantes e número de domicílios no ano de 2010.

\begin{tabular}{cccc}
\hline $\begin{array}{c}\text { REGIÕES } \\
\text { ADMINTRATIVAS } \\
\text { (34 R.AS) }\end{array}$ & $\begin{array}{c}\text { BAIRROS } \\
\text { CORRESPONDENTES }\end{array}$ & $\begin{array}{c}\text { NÚMERO } \\
\text { TOTAL DE } \\
\text { HABITANTES }\end{array}$ & $\begin{array}{c}\text { NÚMERO } \\
\text { TOTAL DE } \\
\text { DOMICÍLIOS }\end{array}$ \\
\hline I PORTUÁRIA & Caju, Gamboa, Santo Cristo e & 48.664 & 17.368 \\
II CENTRO & $\begin{array}{c}\text { Saúde } \\
\text { Centro }\end{array}$ & 41.142 & 22.646 \\
III RIO COMPRIDO & $\begin{array}{c}\text { Catumbi, Cidade } \\
\text { Nova, Estácio, Rio Comprido }\end{array}$ & 78.975 & 28.553
\end{tabular}


Botafogo, Catete, Cosme

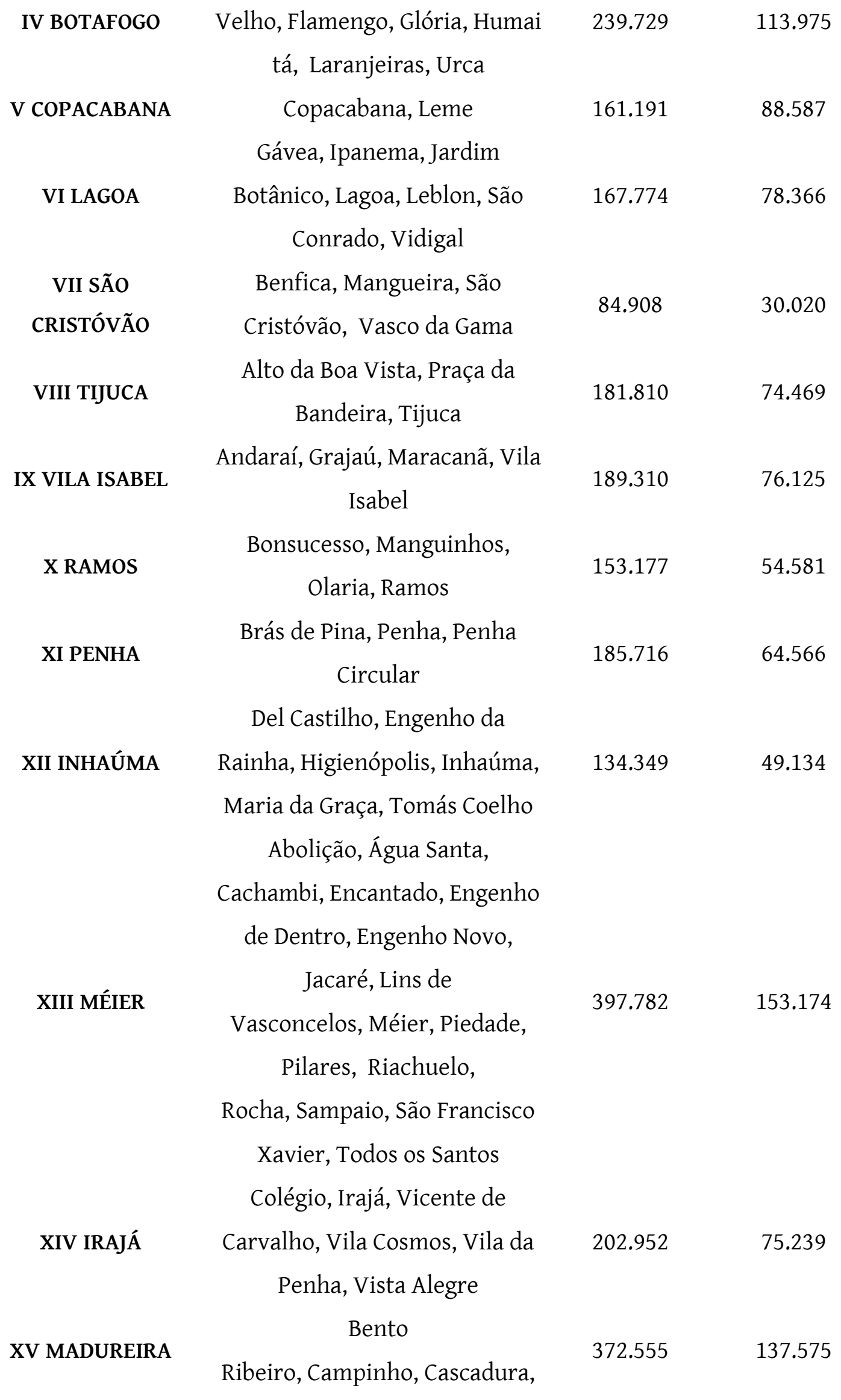


Cavalcanti, Engenheiro

Leal, Honório

Gurgel, Madureira, Marechal

Hermes, Oswaldo

Cruz, Quintino Bocaiúva, Rocha

Miranda, Turiaçu, Vaz Lobo

Anil, Curicica, Freguesia

(Jacarepaguá), Gardênia Azul,

XVI JACAREPAGUÁ Jacarepaguá, Pechincha, Praça

572.030

215.552

Seca, Tanque, Taquara, Vila

Valqueire

XVII BANGU

XVIII CAMPO

GRANDE

Bangu, Gericinó, Padre Miguel, Senador Camará

412.868

143.892

Campo Grande, Cosmos,

Inhoaíba, Santíssimo, Senador

542.084

Vasconcelos

XIX SANTA CRUZ

Paciência, Santa Cruz, Sepetiba

368.534

132.456

Bancários, Cacuia, Cidade

Universitária, Cocotá, Freguesia

(Ilha do Governador), Galeão,

XX ILHA DO

Jardim Carioca, Jardim

Guanabara, Moneró,

Pitangueiras, Portuguesa,

Praia da Bandeira,

Ribeira, Tauá, Zumbi

XXI ILHA DE

PAQUETÁ

Paquetá

3.361

2.223

Anchieta, Guadalupe, Parque

XXII ANCHIETA

Anchieta, Ricardo de

158.318

56.021

Albuquerque

XXIII SANTA

TERESA

Santa Teresa

40.926

16.671 
XXIV BARRA DA

TIJUCA

XXVI GUARATIBA

XXVII ROCINHA

XXVIII

JACAREZINHO

XXIX COMPLEXO

DO ALEMÃO

XXX MARÉ

XXXI VIGÁRIO

GERAL

XXXIII REALENGO

XXXIV CIDADE DE

DEUS
Barra da Tijuca, Camorim,

Grumari, Itanhangá, Joá,

Recreio dos Bandeirantes

300.823

136.743

Vargem Grande, Vargem

Pequena

Acari, Barros Filho, Coelho

Neto, Costa Barros, Parque

208.813

71.537

Columbia, Pavuna

Barra de Guaratiba,

Guaratiba, Pedra de Guaratiba

123.114

48.605

Rocinha

69.356

Jacarezinho

37.839

11.881

Complexo do Alemão

69.143

21.272

Complexo da Maré

129.770

43.038

Cordovil, Jardim

América, Parada de Lucas,

136.171

46.559

Vigário Geral

Campo dos Afonsos,

Deodoro, Jardim Sulacap,

Magalhães Bastos,

243.006

87.406

Realengo, Vila Militar

Cidade de Deus

36.515

12.285

Nota: R.A XXXII, dados não fornecidos.

Fonte: Modificada de: BAIRROS CARIOCAS. Prefeitura da Cidade do Rio de Janeiro, 2010. (http://portalgeo.rio.rj.gov.br/bairroscariocas/index_ra.htm)

\section{Regiões administrativas e a Dengue}


Observa-se na tabela abaixo (Tab.2) que o número de casos de dengue em 2014, na maioria das R.As foi menor do que nos anos 2010 e 2016. Vale ressaltar que neste último ano citado que apresenta os maiores índices da doença, a Cidade sediou os Jogos Olímpicos e diversas obras visando a melhoria da infraestrutura foram realizadas, conforme já citado.

Relacionando os dados desta doença de 2010 e 2014, percebe-se que 11 R.As possuíram um aumento do número de casos, onde no Complexo do Alemão e Bangu este percentual foi consideravelmente maior.

Comparando 2014 com 2016, observa-se que todas as R.As apresentaram um crescimento no número de casos. Chama-se atenção para a R.A de Bangu, com 3.257 casos em 2016. Em relação ao percentual de casos, o maior foi na Cidade de Deus.

Tabela 2 - Número total de casos de Dengue em 2010, 2014 e 2016 nas R.As do Rio de Janeiro.

\begin{tabular}{|c|c|c|c|c|c|}
\hline $\begin{array}{c}\text { REGIÕES } \\
\text { ADMINISTRATI } \\
\text { VAS }\end{array}$ & $\begin{array}{c}\text { TOTAL } \\
\text { DE } \\
\text { CASOS } \\
(2010)\end{array}$ & $\begin{array}{l}\text { TOTAL DE } \\
\text { CASOS } \\
(\mathbf{2 0 1 4 )}\end{array}$ & $\begin{array}{c}\text { PERCENTUAL DE } \\
\text { AUMENTO/REDU } \\
\text { ÇÃO NO NÚMERO } \\
\text { DE CASOS (\%), } \\
\text { PERIODO DE } \\
2010 / 2014\end{array}$ & $\begin{array}{c}\text { TOTAL } \\
\text { DE } \\
\text { CASOS } \\
\text { (2016) }\end{array}$ & $\begin{array}{c}\text { PERCENTUAL } \\
\text { DE AUMENTO } \\
\text { NO NÚMERO } \\
\text { DE CASOS (\%), } \\
\text { PERIODO DE } \\
2014 \text { A } 2016\end{array}$ \\
\hline I-PORTUÁRIA & 46 & 17 & 36 & 253 & 1.488 \\
\hline II-CENTRO & 46 & 24 & 52 & 125 & 520 \\
\hline III-RIO COMPRIDO & 54 & 25 & 46 & 877 & 3.508 \\
\hline IV-BOTAFOGO & 157 & 109 & 69 & 251 & 230 \\
\hline V-COPACABANA & 139 & 46 & 33 & 155 & 336 \\
\hline VI-LAGOA & 117 & 31 & 26 & 161 & 519 \\
\hline $\begin{array}{c}\text { VII-SÃO } \\
\text { CRISTÓVÃO }\end{array}$ & 46 & 32 & 69 & 228 & 712 \\
\hline VIII-TIJUCA & 103 & 116 & 112 & 164 & 141 \\
\hline
\end{tabular}




\begin{tabular}{|c|c|c|c|c|c|}
\hline IX-VILA ISABEL & 112 & 101 & 90 & 814 & 805 \\
\hline X-RAMOS & 85 & 44 & 51 & 723 & 1.643 \\
\hline XI-PENHA & 89 & 118 & 132 & 1.845 & 1.563 \\
\hline XII-INHAÚMA & 51 & 43 & 84 & 727 & 1.690 \\
\hline XIII-MÉIER & 238 & 159 & 66 & 1.424 & 895 \\
\hline XIV-IRAJÁ & 98 & 104 & 106 & 969 & 931 \\
\hline XV-MADUREIRA & 141 & 103 & 73 & 1.414 & 1.372 \\
\hline XVI-JACAREPAGUÁ & 274 & 207 & 75 & 1.896 & 915 \\
\hline XVII-BANGU & 133 & 378 & 284 & 3.257 & 861 \\
\hline $\begin{array}{l}\text { XVIII-CAMPO } \\
\text { GRANDE }\end{array}$ & 283 & 121 & 42 & 983 & 812 \\
\hline XIX-SANTA CRUZ & 141 & 166 & 117 & 1.433 & 863 \\
\hline $\begin{array}{l}\text { XX-ILHA DO } \\
\text { GOVERNADOR }\end{array}$ & 163 & 82 & 50 & 207 & 252 \\
\hline XXI-PAQUETÁ & 10 & 11 & 110 & 57 & 518 \\
\hline XXII-ANCHIETA & 72 & 31 & 43 & 388 & 1.251 \\
\hline $\begin{array}{c}\text { XXIII-SANTA } \\
\text { TERESA }\end{array}$ & 24 & 31 & 129 & 173 & 558 \\
\hline $\begin{array}{l}\text { XXIV-BARRA DA } \\
\text { TIJUCA }\end{array}$ & 189 & 92 & 48 & 906 & 984 \\
\hline XXV-PAVUNA & 141 & 73 & 51 & 848 & 1.161 \\
\hline XXVI-GUARATIBA & 94 & 114 & 121 & 467 & 409 \\
\hline XXVII-ROCINHA & 53 & 19 & 35 & 154 & 810 \\
\hline $\begin{array}{c}\text { XXVIII- } \\
\text { JACAREZINHO }\end{array}$ & 5 & 9 & 180 & 22 & 244 \\
\hline $\begin{array}{c}\text { XXIX- COMPLEXO } \\
\text { DO ALEMÃO }\end{array}$ & 9 & 28 & 311 & 1.268 & 4.528 \\
\hline
\end{tabular}




$\begin{array}{cccccc}\text { XXX- MARÉ } & 11 & 10 & 90 & 48 & 480 \\ \text { XXXIII-REALENGO } & 99 & 113 & 114 & 1.557 & 1.377 \\ \begin{array}{c}\text { XXXIV-CIDADE DE } \\ \text { DEUS }\end{array} & 23 & 3 & 13 & 181 & 6.033 \\ \end{array}$

Nota: R.As XXXI e XXXII, dados não fornecidos.

Fonte: Modificado de Prefeitura da Cidade do Rio de Janeiro (http://www.rio.ri.gov.br/web/sms/dengue-casos-bairro-periodo), 2017.

A tabela abaixo (Tab.3) representa a quantidade (em toneladas) de lixo público que foram coletados nos anos de 2010 e 2014. Estes dados foram coletados a fim de compará-los com os dados referentes ao índice de casos de dengue (Tab.2), uma vez que estes resíduos podem servir de criadouros para os mosquitos.

Nota-se de um modo geral, que a quantidade de lixo público coletado diminuiu em 21 R.As de 2010 para 2014 e apenas em 11, esta quantidade aumentou. A R.A que teve mais lixo coletado em 2014 foi Bangu com 99.386 toneladas. Já em 2010, foi Campo Grande com 114.178 toneladas. As R.As que menos possuíram lixo público para coleta foram Santa Teresa em 2014 e Paquetá, em 2010. Em relação ao percentual de aumento, este foi maior na Cidade de Deus, e o que mais reduziu seu percentual, foi a R.A de Santa Teresa.

Tabela 3 - Total do lixo público coletado por ano, segundo Regiões Administrativas Município do Rio de Janeiro - 2010 - 2014. 


\begin{tabular}{|c|c|c|c|}
\hline $\begin{array}{c}\text { REGIÕES } \\
\text { ADMINISTRATIVAS }\end{array}$ & $\begin{array}{c}\text { LIXO } \\
\text { PÚBLICO (T) } \\
2010\end{array}$ & $\begin{array}{c}\text { LIXO } \\
\text { PÚBLICO (T) } \\
2014\end{array}$ & $\begin{array}{c}\text { \% DO } \\
\text { AUMENTO/REDUÇÃO } \\
\text { DO LIXO PÚBLICO DE } \\
2010 \text { PARA } 2014\end{array}$ \\
\hline I-PORTUÁRIA & 20705 & 17532 & 84 \\
\hline II-CENTRO & 33850 & 14404 & 42 \\
\hline III-RIO COMPRIDO & 20159 & 18348 & 91 \\
\hline IV-BOTAFOGO & 32157 & 26396 & 82 \\
\hline V-COPACABANA & 27909 & 29568 & 106 \\
\hline VI-LAGOA & 21074 & 26767 & 127 \\
\hline VII-SÃO CRISTÓVÃO & 23877 & 39363 & 164 \\
\hline VIII-TIJUCA & 24439 & 20235 & 82 \\
\hline IX-VILA ISABEL & 20090 & 17579 & 87 \\
\hline X-RAMOS & 37908 & 16626 & 43 \\
\hline XI-PENHA & 32998 & 25332 & 76 \\
\hline XII-INHAÚMA & 40797 & 37309 & 91 \\
\hline XIII-MÉIER & 63018 & 56754 & 90 \\
\hline XIV-IRAJÁ & 35563 & 33574 & 94 \\
\hline XV-MADUREIRA & 60689 & 46717 & 76 \\
\hline XVI-JACAREPAGUÁ & 77344 & 55349 & 71 \\
\hline XVII-BANGU & 107165 & 99386 & 92 \\
\hline XVIII-CAMPO GRANDE & 114178 & 85128 & 74 \\
\hline XIX-SANTA CRUZ & 22587 & 38310 & 169 \\
\hline $\begin{array}{l}\text { XX-ILHA DO } \\
\text { GOVERNADOR }\end{array}$ & 39272 & 32095 & 81 \\
\hline XXI-PAQUETÁ & 6715 & 6585 & 98 \\
\hline XXII-ANCHIETA & 24899 & 27768 & 111 \\
\hline XXIII-SANTA TERESA & 9534 & 2946 & 30 \\
\hline XXIV-BARRA DA TIJUCA & 43424 & 44048 & 101 \\
\hline XXV-PAVUNA & 38524 & 49217 & 127 \\
\hline
\end{tabular}




\begin{tabular}{cccc} 
XXVI-GUARATIBA & 13566 & 18248 & 134 \\
XXVII-ROCINHA & 15523 & 11471 & 73 \\
$\begin{array}{c}\text { XXIX- COMPLEXO DO } \\
\text { ALEMÃO }\end{array}$ & 8116 & 9976 & 122 \\
XXX- MARÉ & 36553 & 39902 & 109 \\
XXXI VIGÁRIO GERAL & 23930 & 19151 & 80 \\
XXXIII-REALENGO & 61232 & 53699 & 87 \\
XXXIV-CIDADE DE DEUS & 7186 & 13837 & 192 \\
\hline
\end{tabular}

Nota: R.As XXVIII e XXXII: Dados não fornecidos

Fonte: Adaptada do site Prefeitura da Cidade do Rio de Janeiro (http://www.armazemdedados.rio.r.j.gov.br/)

O quadro abaixo (Quadro 1) apresenta informações referentes à rede de esgoto em todo município do Rio de Janeiro do período de 2000 até 2014. Observa-se que em relação à porcentagem de atendimento total de esgoto, em 2014, este índice foi o segundo melhor durante este período de 14 anos e em 2010, este índice foi o segundo pior. Já em relação aos índices de coleta e tratamento destes efluentes, estes não apresentaram as melhores porcentagens durante o último ano apresentado (2014) o que leva a pensar no motivo para estes índices terem diminuído com o tempo, principalmente para a porcentagem de tratamento de esgoto, apresentando possíveis riscos de contaminação e aparecimento de doenças que podem ser nocivas à saúde do homem.

Quadro 1 - Principais indicadores de prestação do serviço de coleta de esgoto - 
Município do Rio de Janeiro - 2000 à 2014.

\begin{tabular}{|c|c|c|c|c|c|}
\hline № & $\begin{array}{c}\text { ÍNDICE DE } \\
\text { ATENDIMENTO } \\
\text { TOTAL DE } \\
\text { ESGOTO (\%) }\end{array}$ & $\begin{array}{l}\text { ÍNDICE DE } \\
\text { COLETA DE } \\
\text { ESGOTO } \\
(\%)\end{array}$ & $\begin{array}{c}\text { ÍNDICE DE } \\
\text { TRATAMENTO } \\
\text { DE ESGOTO } \\
\text { (\%) }\end{array}$ & $\begin{array}{c}\text { ÍNDICE DE } \\
\text { ESGOTO } \\
\text { TRATADO P/ } \\
\text { ÁGUA } \\
\text { CONSUMIDA } \\
(\%)\end{array}$ & $\begin{array}{c}\text { EXTENSÃO } \\
\text { DA REDE DE } \\
\text { ESGOTO POR } \\
\text { LIGAÇÃO (m } \\
\text { / LIG) }\end{array}$ \\
\hline 2000 & 91,2 & $\ldots$ & $\ldots$ & $\ldots$ & ... \\
\hline 2001 & 81,6 & ... & 60,2 & ... & ... \\
\hline 2002 & 81,9 & 90,9 & 66,3 & 60,2 & 7,8 \\
\hline 2003 & 82,0 & 75,2 & 76,1 & 57,2 & 6,5 \\
\hline 2004 & 81,5 & 75,1 & 82,7 & 62,1 & 6,6 \\
\hline 2005 & 82,9 & 73,8 & 83,7 & 61,7 & 6,6 \\
\hline 2006 & 82,0 & 84,4 & 80,7 & 68,1 & 6,6 \\
\hline 2007 & 70,0 & 71,5 & 83,8 & 59,9 & 7,4 \\
\hline 2008 & 82,4 & 55,8 & 85,3 & 47,6 & 7,2 \\
\hline 2009 & 68,7 & 77,1 & 97,8 & 75,4 & 7,4 \\
\hline 2010 & 70,1 & 61,5 & 86,5 & 53,2 & 7,0 \\
\hline 2011 & 77,9 & 61,5 & 84,4 & 51,9 & 5,8 \\
\hline 2012 & 78,2 & 62,8 & 79,6 & 50,0 & 6,5 \\
\hline 2013 & 80,9 & 65,6 & 71,9 & 47,2 & 6,5 \\
\hline 2014 & 83,1 & 66,2 & 71,3 & 47,2 & 6,6 \\
\hline
\end{tabular}

Fonte: Adaptada do site da Prefeitura da Cidade do Janeiro. (http://www.armazemdedados.rio.rj.gov.br/) Ministério das Cidades- Secretaria Nacional de Saneamento Ambiental- Sistema Nacional de informações sobre Saneamento (SNIS).

\section{DISCUSSÃO}

Em relação à tabela da dengue (Tab.2) chama-se atenção em relação à comparação de números de casos da dengue de 2014 para 2016. Sabe-se que neste último período a Cidade sediou os Jogos Olímpicos e conforme já dito, várias obras foram desenvolvidas visando a infraestrutura da Cidade. Nota-se que em 2016, a R.A de Bangu liderou o ranking quanto ao número de casos da dengue com 3.233 casos, seguido pela R.A de Jacarepaguá, com 1.896 casos o que representou um grande aumento no número de casos de dengue de 2014 para 
2016, não só nestas regiões citadas. Segundo Demarzo et al. (2014) ainda há poucos estudos que apoiam o fato da Cidade sediar as Olimpíadas esteja relacionado com a melhoria na qualidade da saúde da população, ou seja, que há outros fatores que podem influenciar no aumento dessa enfermidade como a falta de conscientização por parte da população na diminuição dos focos de mosquitos vetores da doença.

Ao comparar os casos de dengue com a coleta de lixo público, observa-se que em 2014, a quantidade de lixo público coletado diminuiu em 20 R.As, assim como no número de casos da dengue (20), possuindo 13 R.As em comum (vale lembrar que as R.As de Jacarezinho, Maré e Vigário Geral não foram comparadas por possuírem dados incompletos de dengue e coleta de lixo). Uma suposição leva a crer numa possível redução da produção de lixo público e desta forma, menos criadouros de mosquito teriam surgido. Porém, segundo Carneiro Neto e Pinheiro (2016, p.26) a Cidade do Rio de Janeiro foi apontada como sendo "uma das mais sujas", onde "o estado recolheu 1.225.690 toneladas de resíduos em 2012". De acordo com este fato, isto impossibilita a crer que houve uma diminuição na produção de lixo, já que o consumo humano é influenciado cada vez mais pelo desenvolvimento das indústrias.

Comparando os índices de atendimento total de esgoto com os números de casos de dengue nos anos de 2010 e 2014, observa-se que em 2010, este atendimento foi um dos piores nestes 14 anos e em relação à dengue, este ano apresentou o segundo maior índice da doença em relação aos anos analisados (2010, 2014 e 2016). Em 2014, os índices desta doença na maioria das R.As foram menores em relação à 2010 e houve uma melhoria do atendimento total de esgoto.

Carvalho; Magalhães e Medronho (2017) afirmam que a insuficiência de saneamento básico é capaz de contribuir para aparecimento da doença, onde as regiões mais quentes e próximas às comunidades apresentam um quadro de maior vulnerabilidade social (principalmente devido à falta de infraestrutura e serviços básicos), o que serve como um fator 'atrativo' para a dengue.

Porém, não se pode afirmar que estes fatores são totalmente influenciadores dos índices de dengue, embora possam contribuir para o aparecimento da doença. Ressalta-se então, que outros fatores devem ser considerados como os fatores ambientais e 
meteorológicos como o clima e a temperatura de cada região, conforme afirmado por Gomes; Nobre; Cruz (2012), que podem influenciar na disseminação do patógeno e favorecer a permanência destes vetores. Segundo Soares; Fragoso; Cruz (2017) existem condições favoráveis para a geração de criadouros em regiões ricas e pobres, além da temperatura, que por ser mais elevada no mês de fevereiro, aumenta o número de fêmeas de Aedes aegypti, além de fatores que de forma combinada podem contribuir para que os casos de dengue possam ter um aumento significativo.

\section{CONCLUSÃO}

A questão sanitária há muito tempo é um tema que ainda causa grandes preocupações na Cidade do Rio de Janeiro, principalmente porque a sua insuficiência é responsável pela acentuação de diversas doenças e problemas urbanos, conforme observados durante os séculos XIX e início do XX. Com a revolta da vacina e a urbanização da cidade neste período, além da criação de leis em prol do saneamento básico durante os anos 90 , era esperado que o perfil da cidade melhorasse e que este quesito fosse valorizado.

Ainda hoje são encontradas algumas ineficiências dos serviços básicos de infraestrutura e saúde, embora neste último ano de 2016, diversas obras tenham sido feitas visando a melhoria destes serviços na cidade. Contudo, tais dificuldades podem não ser as únicas causas relacionadas à veiculação de doenças, principalmente da dengue. No Município do Rio de Janeiro, as R.As apresentam diferenças ligadas às questões de desigualdade social e urbanização, que devem ser levadas em conta e consideradas como influentes já que impactam no crescimento e disseminação de doenças veiculadas por mosquitos cujo crescimento e dispersão são influenciados diretamente por fatores como clima e temperatura.

\section{REFERÊNCIAS}


BAIRROS CARIOCAS. Prefeitura da Cidade do Rio de Janeiro, 2010. Disponível em:< http://portalgeo.rio.rj.gov.br/bairroscariocas/index_ra.htm> Acesso em 23 de abril de 2017.

BAIRROS CARIOCAS, Prefeitura da Cidade do Rio de Janeiro, Estatísticas municipais- Infraestrutura-Esgoto e drenagem, 2014. Disponível em:< http://www.armazemdedados.rio.ri.gov.br/> Acesso em 16 de maio de 2017.

BAIRROS CARIOCAS, Prefeitura da Cidade do Rio de Janeiro, Estatísticas municipais- InfraestruturaResíduos sólidos, 2014. Disponível em:< http://www.armazemdedados.rio.rj.gov.br/> Acesso em 16 de maio de 2017.

BRASIL. Lei $n^{\circ} 8.080$ de 19 de Setembro de 1990. Dispõe sobre as condições para a promoção, proteção e recuperação da saúde, a organização e o funcionamento dos serviços correspondentes e dá outras providências. Diário Oficial da União - Seção 1 - 20/9/1990, Página 18055, 1990. Disponível em:< http://www.planalto.gov.br/ccivil_03/leis/L8080.htm> Acesso em 15 de abril de 2017.

BRASIL. Lei № 11.445, de 5 de Janeiro de 2007. Estabelece diretrizes nacionais para o saneamento básico; altera as Leis $\mathrm{n}^{\text {os }} 6.766$, de 19 de dezembro de 1979, 8.036, de 11 de maio de 1990, 8.666, de 21 de junho de 1993, 8.987, de 13 de fevereiro de 1995; revoga a Lei ${ }^{\circ} 6.528$, de 11 de maio de 1978; e dá outras providências. Diário Oficial da União - Seção 1 - 8/1/2007, Página 3, 2007. Disponível em: $<$ https://www.planalto.gov.br/ccivil_03/_ato2007-2010/2007/lei/l11445.htm> Acesso em 15 de abril de 2017.

BRASIL, Lei $\mathrm{n}^{\mathrm{0}}$ 12.305 de 2 de Agosto de 2010. Institui a Política Nacional de Resíduos Sólidos; altera a Lei no 9.605, de 12 de fevereiro de 1998; e dá outras providências. Diário Oficial da União - Seção 1 - 3/8/2010, Página 3, 2010. Disponível em: < http://www.mma.gov.br/port/conama/legiabre.cfm?codlegi=636> Acesso em 15 de abril de 2017.

CAMPOS, C. E. A; COHN, A; BRANDÃO, A. L. Trajetória histórica da organização sanitária da Cidade do Rio de Janeiro: 1916-2015. Cem anos de inovação e conquistas. Ciência e Saúde Coletiva, vol.21, n.5, p.1351-1364, 2016. Disponível em:< http://www.scielo.br/pdf/csc/v21n5/1413-8123-csc-21-05-1351.pdf> .Acesso em 15 de abril de 2017.

CARNEIRO NETO, A.F; PINHEIRO, R. B. M. Coleta de resíduos no Estado do Rio de Janeiro: Desafio e comprometimento público. R. gest. sust. ambient. v. 4, n. 2, p. 489-529, 2016. Disponível em: http://www.portaldeperiodicos.unisul.br/index.php/gestao_ambiental/article/view/3222/2290. Acesso em 30 de maio de 2017.

CARVALHO, J.C.B; ORSINE, J.V.C. Contaminação do meio ambiente por fontes diversas e os agravos a saúde da população. ENCICLOPÉDIA BIOSFERA, Centro Científico Conhecer - Goiânia, v.7, n.13, p.1107-1118, 2011. Disponível em:< http://www.conhecer.org.br/enciclop/2011b/ciencias\%20ambientais/contaminacao.pdf>. Acesso em 13 de março de 2017.

CARVALHO, S; MAGALHÃES, M.A.F.M; MEDRONHO, R.A. Análise da distribuição espacial de casos da dengue no município do Rio de Janeiro, 2011 e 2012. Rev. Saúde Pública, v.51, 2017. Disponível em:< http://www.fsp.usp.br/rsp/artigo/analise-da-distribuicao-espacial-de-casos-da-dengue-no-municipio-do-riode-janeiro-2011-e-2012/>. Acesso em 14 de setembro de 2017.

DEMARZO, M.M.P; MAHTANI, K.R; SLIGHT, S.P; BARTON, C; BLAKEMAN, T; PROTHEROEJ. Legado olímpico para o Brasil: questão de saúde pública?. Cad. Saúde Pública, Rio de Janeiro, v.30, n.1, p.8-10, 2014. Disponível em: $<$ http://www.scielo.br/pdf/csp/v30n1/0102-311X-csp-30-01-00008.pdf>. Acesso em 16 de abril de 2017.

DORNELLES, B; MARTINS, M.N. Rio 2016: zika vírus e a defasagem noticiosa entre o on-line e o impresso no agendamento das olimpíadas do Brasil. LOGOS 44 Dossiê: Mídia, Esporte e Cultura. v.23, n 01, p. 134-146 2016. Disponível em $:<$ http://www.e-publicacoes.uerj.br/index.php/logos/article/view/22263>. Acesso em 16 de abril de 2017.

GOMES, A.F; NOBRE, A.A; CRUZ, O.G. Temporal analysis of the relationship between dengue and meteorological variables in the city of Rio de Janeiro, Brazil, 2001-2009. Cad. Saúde Pública. v.28, n.11, p. 2189-2197, 2012. Disponível em:< http://www.scielo.br/scielo.php?script=sci_arttext\&pid=S0102-311X2012001100018 >. Acesso em 13 de fevereiro de 2017. 
LIMA, E.C.R. Qualidade da água da Baía de Guanabara e saneamento: Uma abordagem sistêmica. Tese de doutorado, Depto. de Engenharia, Universidade Federal do Rio de Janeiro, p.01-183, 2006. Disponível em: < http://www.ppe.ufrj.br/ppe/production/tesis/limaecr.pdf> Acesso em 24 de março de 2017.

MARQUES, E.C. Da higiene à construção da cidade: O Estado e o saneamento no Rio de Janeiro. História, Ciências e Saúde- Manguinhos. $\quad$ v.2, $\quad$ n.2 $\quad$ p.51-67, 1995. Disponível em: < http://www.scielo.br/pdf/hcsm/v2n2/a04v2n2.pdf>. Acesso em 05 de junho de 2017.

MIRANDA, M.A.P; MIRANDA, L.A.P; PICANÇO, M.R.A. O direito ao saneamento como fator social de saúde no Brasil. Cad. IberAmer. Direito. Sanit., v.2, n.2, p. 252-263, 2013. Disponível em:< http://publicaciones.fmdv.org/ojs/index.php/cuadernosderechosanitario/article/view/42/65>. Acesso em 15 de abril de 2017.

OMS. Organização Mundial da Saúde. Health topics- Sanitation, 2017. Disponível em:< http://www.who.int/topics/sanitation/en/ >. Acesso em: 15 de maio de 2017.

PÔRTO, A; PONTE, C.F. Vacinas e campanhas: as imagens de uma história a ser contada. História, Ciências, Saúde- Manguinhos. $\quad$ v.10: 725-742, 2003. Disponível em: < http://www.scielo.br/pdf/hcsm/v10s2/a13v10s2.pdf>. Acesso em 05 de junho de 2017.

REIS, F.B. Análise espacial do saneamento ambiental no território de Manguinhos e seus impactos na saúde da população. Biblioteca Virtual de Saúde Pública Brasil, Fundação Oswaldo Cruz, Rio de Janeiro, p.173, 2016 Disponível em:< http://saudepublica.bvs.br/pesquisa/resource/pt/int-4822> Acesso em 13 de março de 2017.

REIS, D.A ; ESPERIDIÃO, F; JORGE, M.A; RIBEIRO, L.C.S; MOTA, T.S; SANTOS, J.C.N; SILVA, A.T. Estudo bibliométrico da produção científica nacional e internacional no setor de saneamento. Revista GEINTEC, v.7, n.1, p.3669-3685, $2017 . \quad$ Disponível em: http://www.revistageintec.net/portal/index.php/revista/article/view/1077. Acesso em 15 de abril de 2017.

SILVA, C.H. da; LAGE, S.R.M; GUIMARÃES, M.C.S. Produção científica em dengue: um olhar a partir da coleção Brasil da ScielO. In: Encontro Nacional de Pesquisa em Ciência da Informação, 15, p. 5336-5354, 2014. Disponível em:

http://repositorios.questoesemrede.uff.br/repositorios/bitstream/handle/123456789/2764/Produ\%C3\%A7\%C 3\%A30\%20cient\%C3\%ADfica.pdf?sequence=1>. Acesso em 22 de abril de 2017.

SOARES, M; FRAGOSO, V.M.S; CRUZ, F.A.O. Distribuição temporal de dengue no Rio de Janeiro, 1987-2015: Análise e previsão. Scientia Amazonia, v.6, n.2, p. 74-82, 2016. Disponível em: https://www.researchgate.net/publication/315706334_Distribuicao_temporal_de_dengue_no_Rio_de_Janeiro _1987-2015_Analise_e_previsao_Resumo. Acesso em 31 de maio de 2017.

TAUIL, P.L. Urbanização e ecologia do dengue. Cad. Saúde Pública. v.17, p.99-102, 2001. Disponível em: http://www.scielosp.org/pdf/csp/v17s0/3885.pdf. Acesso em 23 de abril de 2017.

VALLE, D; PIMENTA, D.N; AGUIAR, R. Zika, dengue e chikungunya: desafios e questões. Epidemiol. Serv. Saude, Brasília, v.25, n.2, p.419-422, 2016. Disponível em: http://scielo.iec.pa.gov.br/pdf/ess/v25n2/22379622-ess-25-02-00419.pdf Acesso em 22 de abril de 2017.

WERMELINGER, E.D; CARVALHO, R.W de. Métodos e procedimentos usados no controle do Aedes aegypti na bem-sucedida campanha de profilaxia da febre amarela de 1928 e 1929 no Rio de Janeiro. Epidemiol. Serv. Saude, Brasília, v.25, n.4, p. 837-844 2016. Disponível em :< http://www.scielo.br/pdf/ress/v25n4/2237-9622ress-25-04-00837.pdf> Acesso em 22 de abril de 2017. 